\title{
Study of Effectiveness of Operator Training Simulators in the Oil and Gas Industry
}

\author{
Kallakuri Ravikanth ${ }^{1}$, Dr. P.C. Bahuguna ${ }^{2}$, Donald C. Glaser ${ }^{3}$, Dr. Sanjay Shivalkar ${ }^{4}$ \\ ${ }^{1}$ Research Scholar- PhD (Oil \& Gas Management), University of Petroleum and Energy Studies, Dehradun, India \& \\ Training Specialist, Dolphin Energy Limited, Doha, State of Qatar. E-Mail: foralldata @ hotmail.com ${ }^{2}$ Associate \\ Professor, University of Petroleum and Energy Studies, Dehradun, India. E-Mail: bahuguna @ddn.upes.ac.in \\ ${ }^{3}$ President, Simulation Solutions Inc., New Jersey, USA. E-Mail: dglaser@ simulation-solutions.com ${ }^{4}$ Senior Training \\ Specialist, Dolphin Energy Limited, Doha, State of Qatar. E-Mail: mitayash@ hotmail.com
}

\begin{abstract}
Control room operators remotely monitor the process data coming from the field, guide field operators and take corrective actions using highly automated and complex distributed control systems. Operators have to intervene during abnormal conditions in the process and bring operations to safe conditions. Human errors remains the top contributor for industrial losses and the challenge is to prevent or address them by training and competency development. Operator training simulators are widely used in the industry to provide hands-on training to operators. Carefully analyzing simulator training effectiveness by predefining standard evaluation models in advance is one method to address the success of the training. Simulator training provides an understanding of the different process logical relationships in order to be able to accurately identify and quickly respond to problems before they escalate. In the current low oil price environment, organizations are facing number of challenges in developing exceptional workforce to sustain the business. The findings of this study re-emphasizes the need to develop training evaluation models integrated with the simulator configuration to effectively analyze the desired outcomes of each training session. This might further contribute to the reduction of operational errors in the hydrocarbon industry.
\end{abstract}

Key words: Control room operators, Human errors, Simulation training, Operator training simulator, Training effectiveness.

\section{Introduction}

The oil and gas industry is meeting the world's growing energy needs in an environmentally and socially responsible way in partnership with different governments and local communities as per international, local and environmental regulations (British Petroleum Outlook, 2017). An expanding population, economic growth, new technology development and scope of regulations transforming the energy landscape of the world. Industrial growth, retiring and ageing workforce, an influx of new workers mean that the organizations must get new employees trained and competent as quickly as possible to sustain in the current low price environment.

\subsection{Human errors in process control}

Process plant operators control, monitor, operate and maintain the plant machinery and equipment during the production and different unit operations. They are able to navigate through piles of DCS (Distributed Control Systems) graphic pages, monitor hundreds of process parameters, make corrective actions and handle abnormal conditions. The ever increasing automation in the industry leading to more sophisticated processes that are more complex for the operators to understand. Consequently, the more sophisticated processes have a tendency to reduce human reliability (Wiener et.al, 1989). The implementation of new technology and automation brings changes to the interface design in control rooms and in the field. This brings to fore possible acclimatization issues faced by workers who may have a high level of familiarity with legacy systems from their experience.

The Center for Chemical Process Safety (CCPS) of American Institute of Chemical Engineers (AIChE), observed that in many incidents, the employees are not fully prepared for a major emergency or incident at the facility and they are not having any formal training on the plant control systems (www.hydrocarbonprocessing.com). The Marsh/McLennan compilation report for business impact shown in Figure 1. shows the losses in 
hydrocarbon sector breaking up into different reasons. It points out that, over $28 \%$ are from operational errors and about $40 \%$ of abnormal operations are caused by human errors.

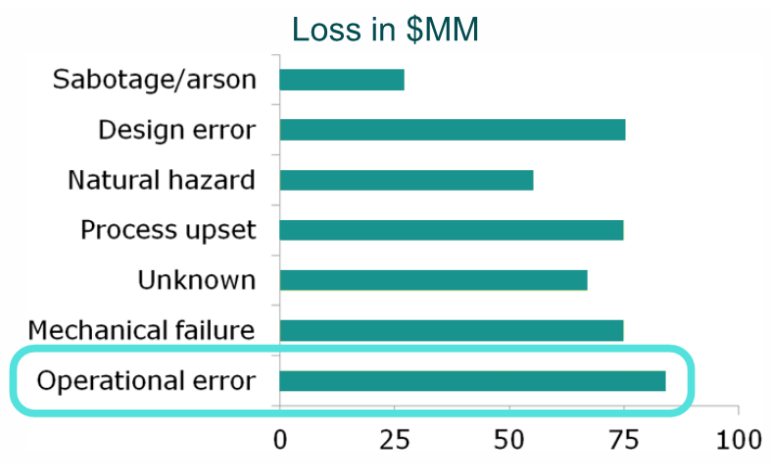

Source: Chemical Safety Topical Committee sponsored by the Department of Energy (DOE)

Figure 1. Causes \& Losses in the Industry

The "abnormal" situations, in particular during the start-up and turn-around, or unscheduled shut-down, are subject to a very substantial part of the significant incidents occurring in many plants. The recurring accidents in the process industry ae due to lack of learning from earlier incidents and applying the lessons learnt from the accidental data and reports.

\subsection{Role of operator training simulator}

Simulators are used for instruction and training in areas such as commercial and military aviation, battle field management, chemical industries, building construction and first responder agencies. They provide a safe alternative in replicating the high-risk, real-world training scenarios. Real world renditions of these situations are too costly and dangerous to provide opportunities for staff to repeatedly practice in the high stress workplace situations (Tichon et al., 2003).

Training simulators are used in the oil and gas industries to train the operators for both routine operations, startup, shutdown and abnormal situations related to the process. The benefits are repeated training on rare events, process upsets, validate or test new procedures, practice startup etc., which in turn increases confidence, enhances their effectiveness and decreases work-related stress. The difference between the operator response with and without appropriate training compiled is in Figure 2. It shows that the response skills are dropping with time after the initial training and practice, typically during the commissioning of the units (www.invensys.com). Response skills of the operators can be sustained with proper training of the simulated scenarios and practice them repeatedly using a suitable training simulator.

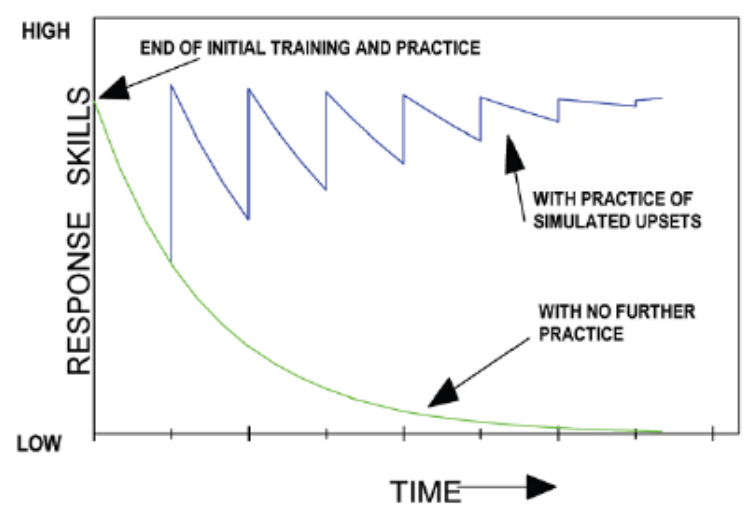

Figure 2. Operator response with and without appropriate training

The confidence in simulators might be due to the fact that most research on their effectiveness has been based on the subjective evaluation of trainees rather than on objective performance data (Salas-2006). The overall goal of training via simulation is to build confidence in staff in their own ability to perform under adverse conditions (Stetz et al., 2006). Due to the advent of modern automation and control systems, operators have less autonomy because they are driven by routine equipment operations, performance and further operators have less opportunity to experience abnormal situations and those situations can be more complex than is the case for manual operation (Hans-2015). A major perception among the managers is that a simulator is a luxury that cannot be justified in financially hard times.

To know that the training is effective, the program needs clear evaluation criteria and process for evaluating the impact on the performance of specific individuals by testing and observing the feedback using defined methodology (Peter-1997). With many similarities to the military, medical and aviation industry, the performance and effectiveness research in these sectors can be applied to the oil and gas industry process control (Kindervarter-2014). The evaluation of training effectiveness is the final stage of developing an effective simulation training program. Process training specialists did not know the impact of their simulator training programs on the effectiveness of individuals or operations teams (Peter-1997) as most of them are not formally trained on evaluation techniques and behavioral based methods. 


\section{Summary of Literature Review}

Training is defined as the planned learning experience that trains the employees how to perform the current and future jobs. Workplace learning improve the profitability of different departments while cultivating more positive attitudes toward profit orientation across an organization. Mostly the training is focused on what needs to be known, rather than the application of skills. Further technological support is one of the most critical support factors to the transfer of learning (Yamnill-2001). The objectives of training determine the most appropriate criteria for assessing the effectiveness of training (Winfred et.al, 2003). The trainee's capabilities, personality, motivational factors, previous work experience will affect the learning process. Training of non-technical skills may help reduce the risk of human error and trained personnel are more prepared to react in case of an emergency situation. The main goal of training is to prepare participants for the tasks they are going to perform on their jobs (Barnard et.al, 2001).

Learning is the process of acquiring the ability to respond adequately to a situation which may or may not have been previously encountered. This means it is a change that occurs in our behaviour (more specifically in our work behaviour) as a result of some experience or practice. The training must focus on this change in behaviour, which gets reflected in performance (Raja-2010) Therefore any training (technical or behavioral) must focus on making the experience a learning one rather than just an enjoyable event. They must be motivated and willing to engage completely in the learning process through a "reflective self-discovery" in order to recognize weaknesses in decision-making and clinical reasoning skills and to be humble enough to correct those weaknesses with the guidance of the instructor (S. Yamnill-2001).

Evaluation is considered as an ongoing process, rather than done only at certain intervals. A number of barriers exist that prevent organizations from evaluating operators at various levels. The three major reasons to evaluate training programs are i) to improve the program, ii) to maximize transfer of learning and achieving subsequent organizational goals and iii) to demonstrate the value of training to the organization, which is effectiveness. In addition to monetary benefits, most training programs will have intangible, non-monetary benefits and the key challenge is to monitor and identify them. When the trainee experiences success or a sense of mastery, it translates into being less aroused physiologically, less distracted and more likely to focus on the task.
Variable-priority training led to better multitasking performance, and a trend for a reduction in the automation complacency effect (Raja-2010).

\subsection{Effectiveness of OTS Training}

Measuring effectiveness is the process of determining whether or not the desired results have been produced. Opinion-based surveys, questionnaires, ratings and checklists are best used in combination with objective measurements (e.g., time, speed, error) of trainee (learning) performance. There is significant change in seafarer attitude, skill level and knowledge due to the usage of simulation training in the shipping industry for different tasks in operations and Kirkpatrick's model is most suitable model for measuring the simulator training effectiveness (Surender-2015). A simulator does not train; it is the way the simulator is used that yields the benefit and the desired results. What is more important than the simulator technology is that how educational methodology is applied and whether it increases training effectiveness significantly or not (Drown-1995).

The simulation literature lacks significant research how the different elements of simulation training can be used to create a learning environment that encourages active learning and engagement in the work place. The training evaluation and effectiveness on the individuals or across operations teams is not studied in the perspective of an operator across the industry using available models. With many similarities with the military, medical, transport and aviation industries, the simulation training effectiveness research in these sectors can be applied in the oil \& gas industry.

\section{Research Methodology and Design}

For the purpose of training assessment, the causeand-effect relationships of interest are those between the process, learning outcome and its impact on training. In these relationships, the process variables (e.g., training methods and mediums used) are indicators of the outcomes, for example, knowledge gained among trainees, learning from incidents and competency etc. The key to identify the essential elements of effective training lies in understanding the correlation of these variables with the intended impact of training (Cohen \& Colligan-1998).

The research objective is to measure the effectiveness of simulation training using proven methods from the literature and apply the methodology to some training sessions in the industry to validate the findings. Based on the literature review and subsequent gap and factor analysis, different 
research questions are formulated as, what is the effectiveness of the operator training using simulators, how to evaluate the effectiveness and really is there a change in knowledge, attitude and skill levels after training and the knowledge acquired being used in work place.The first step to show the value of an training program is to create a program that has organizational value. Based on the review of literature and study of different models Donald Kirkpatrick's Four Level Training Evaluation Model, shown in Figure 3. is adapted as the base model. Kirkpatrick's framework consists of four levels of evaluation. Reaction (Level 1) is a measure of how participants react to the training program and it is a measure of customer satisfaction. Learning (Level 2) is measuring the knowledge gained during the program. Job behavior (Level 3) is measuring how well the participant applies the new knowledge or skills back on the job. This level of evaluation is important as it addresses the issue of training transfer. Level 3 evaluations often show that even though learning took place (Level 2), the skills are seldom fully applied back on the job (Robinson, 1998).

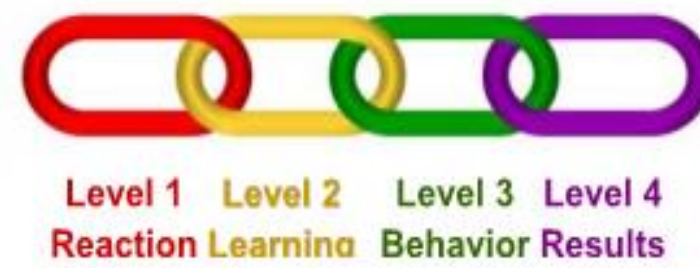

Figure 3. Kirkpatrick's Training Evaluation Model (https://www.kirkpatrickpartners.com/OurPhilosophy/The-Kirkpatrick-Model).

Conducting a Level 3 evaluation can help uncover the reasons that participants do not apply the new skills on the job. Results (Level 4) reflects the evaluation of training's impact on the organization's business results. At this level of evaluation, questions regarding improvement on the organization are answered. The model also assumes that each level is important and that all levels are interlinked. Most importantly organizations change in many ways, and behaviors and results change depending on these as well as on training.

Survey method is used as the primary data gathering tool by using structured questionnaire. Different questionnaires are prepared based on the four levels of Kirkpatrick's evaluation model and the simulator training participants are asked to respond accordingly. The questions are validated by peer review and focused group discussion between five different training specialists in the oil and gas industry. The participants experience is ranging from 10-30 years with an age bracket of 30 to 56 years. Some of the questions have multiple answers and participants are asked to choose what is relevant to them. The central tendency is analyzed to derive the results (P.A. Bishop -2015). Some of the questions are marked using five point Likert's scale, consisting of the scale range is $1=$ strongly disagree, $2=$ disagree, $3=$ neither agree nor disagree, $4=$ agree and $5=$ strongly agree with the same weighted score. The weighted mean score of the data is calculated as below.

[(number of people who selected response 1 )*(weighting of response 1$)+$ (number of people who selected response 2 )*(weighting of response 2)... (Number of people who selected response $\mathrm{n}$ )*(weighting of response $\mathrm{n}$ )] / (total number of respondents)

The survey is carried out during the months February-March 2018 at three sites consisting of around fifty (corresponding to an population of 100, precision level of $\pm 10 \%$, (Smith, 1983)) respondents using two different training simulators supplied by two different OTS. The simulated processes include Heat Exchangers, Distillation, and Natural Gas processing units with the different processing equipment modelled along with the process shutdown systems similar to the operating plant. The demographic data is kept confidential along with the names of the companies as per the internal agreement. The results are analyzed and presented in the next section and the corresponding questions are presented in sub-sections.

\section{Findings from the Survey}

Training effectiveness will depend on a number of factors, such as time spent, motivation, learning efficacy and cognitive ability. Behavioral based standard assessment of operators is more important to mitigate the risk of human error and needs to be integrated with the modern simulators as a standard option, which will reduce the dependency on the instructor and same can be applied to all training sessions using the same simulator. The four levels of evaluation is summarized as below.

\subsection{Reaction}

Based on the survey results in Figure 4. we can conclude that prebriefing is necessary to give a brief idea of planned training sessions and the limitations of the training models used for training. Prebriefing 
increases the confidence of trainees and it was overall beneficial to them.

\subsubsection{Questionnaire}

Q1. Prebriefing increased my confidence and was beneficial to my overall learning experience

Q2. Prebriefing is necessary to know what is planned in the exercise and the simulator limitations.

Q3. I have lost interest during the prebriefing as we already know what we have to do during the exercise. Q4. Prebriefing is not required as we already know what is in the simulation training.

Q5. We need to update the simulators continuously in tandem with the real plant to have updated model for training.

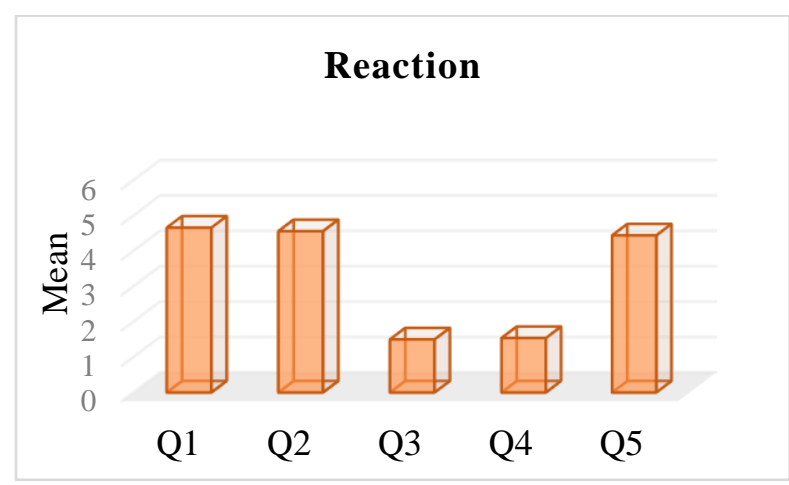

Figure 4. Reaction level

Some of the operators mentioned that prebriefing is not required, as they already know what is expected of them during the training. More than $90 \%$ of operators responded that the skills learned in the simulation training reflected in the actual happenings in the job and repetitive training helped them to handle the situations. Some of the low scores are related directly to the low level of experience of some operators responding to the survey. Majority of the respondents agreed that the OTS models to be updated simultaneously with the plant so that they can practice before the actual implementation of control loops in the OTS and refresh their skills.

\subsection{Learning}

All the questions related to learning are rated highly by the participants as captured in Figure 5. The simulator training directly related to their job responsibilities and participants are motivated to learn new things due to this similarity. Some of them mentioned that they were confident of troubleshooting and understanding of the complex control loops better than the theoretical sessions in the class room. This concludes that the current training helped them to develop a better understanding of the process control and exposure to the critical operations. OTS also gave an opportunity to trainees to practice their decision making skills in a controlled atmosphere and gain confidence in executing the tasks.

\subsubsection{Questionnaire}

Q1. I am better prepared \& respond to changes in process conditions.

Q2. I developed better process control and exposure to critical operations.

Q3. I gain more confidence in trouble shooting.

Q4. I can make critical interventions using complex control loops.

Q5. I am more confident to contribute for process optimization.

Q6. I had the opportunity to practice my decision making skills.

Q7. I am more confident to prioritize operations.

Q8. I learned from observing my peers and actively involved due to collective learning.

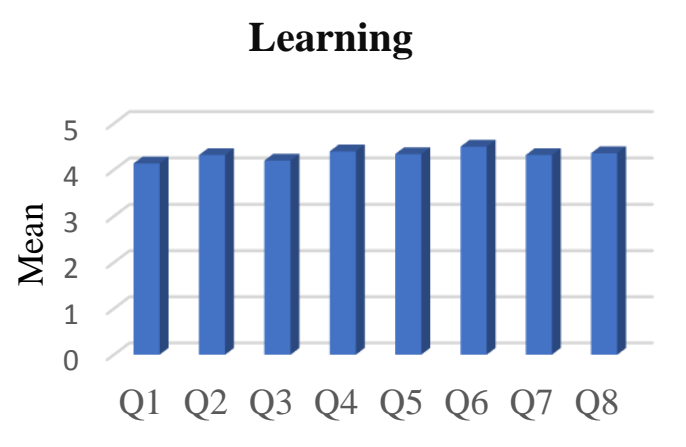

Figure 5. Learning level

The results from learning level emphasizes the trainee's motivation, behavioral strength and confidence in executing different tasks during the actual operations after the training. Interaction with other peers during the training helped them to use the OTS as an observational tool to gain confidence and motivated to make their workplace better.

\subsection{Behavior}

There is significant change in the behavior of the participants during and after the training as noted by the instructor and is further envisaged from the feedback. The results compiled in Figure 6. provided an opportunity to introspect individual performance. 


\subsubsection{Questionnaire}

Q1. Debriefing contributed to my learning and was valuable in helping me improve my judgment.

Q2. Debriefing allowed me to verbalize my feelings before focusing on the next scenario.

Q3. Debriefing provided opportunities to introspect my performance during simulation.

Q4. I am more confident in communicating with my supervisors \& engineers during critical operations.

Q5. I am more confident to train \& can be able to guide others in the control room operations

Q6. Debriefing was a process of constructive evaluation of the simulation session.

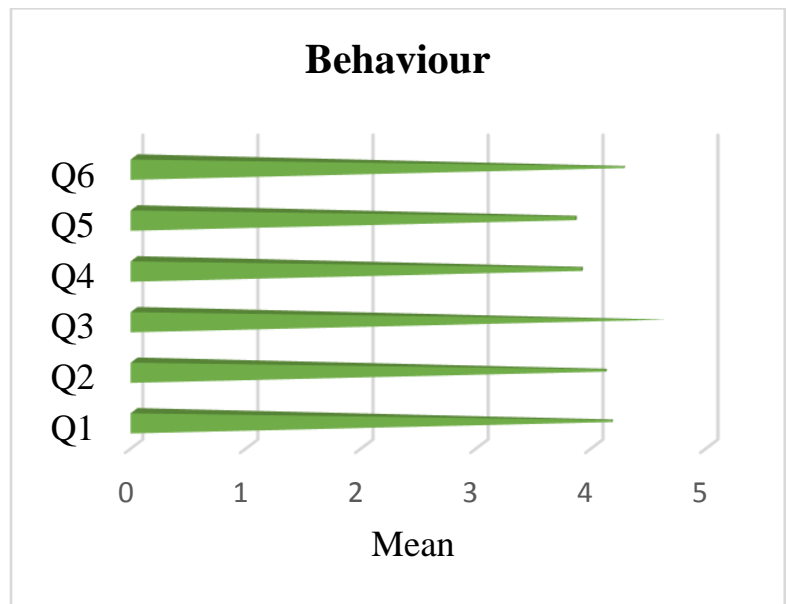

Figure 6. Behavior level

The discussions during simulation sessions helped them to communicate with their superiors in more efficient way and work as a team to further consolidate their learning. During critical operations they are more confident to act and handle the process upsets and might seek opinion with support staff to achieve desired results. The participants considered debriefing as a constructive evaluation of the simulation session. The confidence gained during the training is useful to guide others in the panel operations after practicing repeated sessions using a simulator.

\subsection{Results}

Most of the participants believe that simulation training helped them to understand the process in a better way. Further the practice sessions helped their ability to integrate the theoretical knowledge in to practical experience. The OTS investment can be supported by the reduction of human errors, production quality improvements, performance improvement and the most rated being the startup \& shutdown without incidents. The questionnaire and their corresponding answers Figure 7. are compiled as below.

\subsubsection{Questionnaire}

Q1. This training can be used in all process operator trainings.

Q2. The knowledge acquired is used in the workplace effectively.

Q3. I am more confident in using evidence based practice and hands on approach in control room operations.

Q4. I am more confident in carrying out interventions that foster operational safety.

Q5. There is significant change in application of skills, attitude and confidence levels before and after simulation training.

Q6. I am more confident in critical control loops, advanced process control and optimization due to experience gained from simulated operations.

Q7. Simulator training is the most effective training for operators among all other types of training available in the Oil \& Gas Industry.

Q8. Simulation evaluation can be effectively used as a competency testing tool for promotions and career planning.

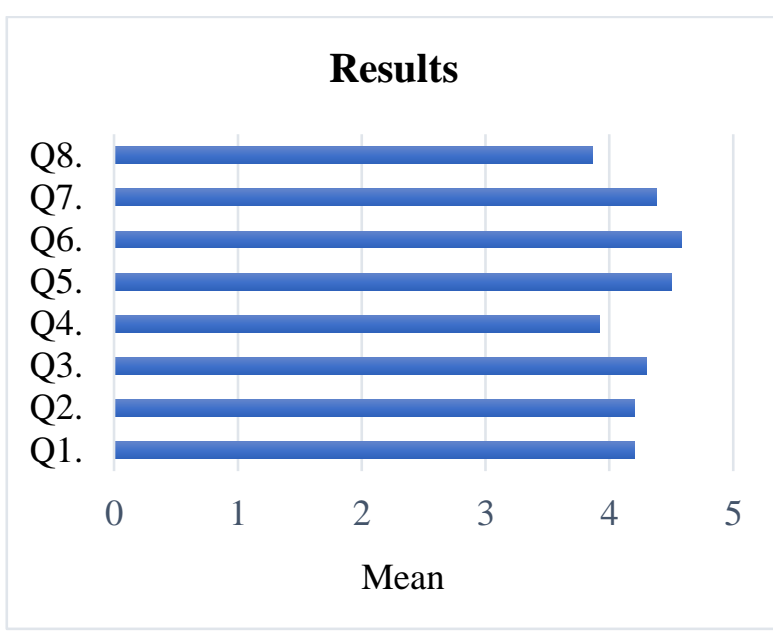

Figure.7. Results level

As per the results, the simulation sessions should be planned soon after the theoretical class room sessions to have effective learning and hands on application. Well planned simulation sessions using updated simulator voted in Figure 8. For maximum utilization of the OTS. The qualified instructors preferably from the same domain experience can help the trainees to run scenarios, which are actually happened in their process operations. They can be 
taken from the incident reports and corrective action reports or similar case studies developed in the simulator, as an exercise preferably by the OTS supplier.

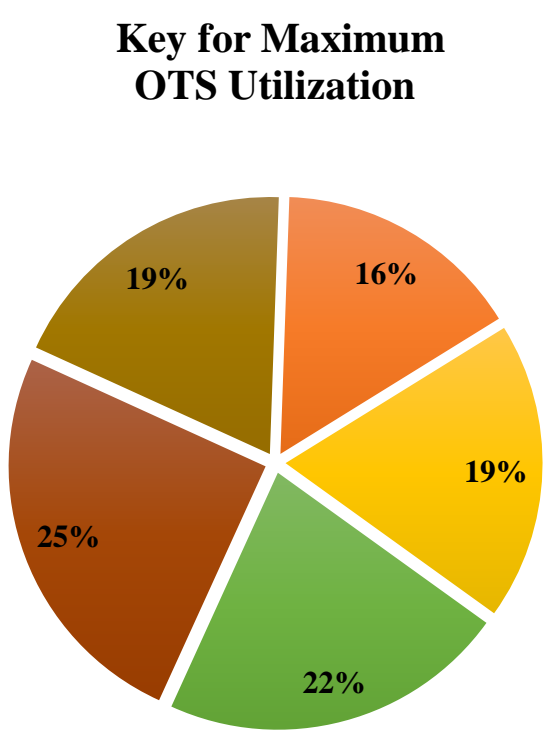

- Support from Management

Qualified Instructors with same domain experience

- Up to date Simulator Model

- Classroom training followed by Simulation training

Well planned and executed training sessions

Figure 8. The Key for successful utilization of simulation training

The operators are better prepared to handle the process upsets, reduction of human errors Figure 9. By replicating the lessons learnt during actual panel operations and, safer startups. This will effectively justifies the OTS investment for the organization. The OTS training sessions helped participants to analyze the process better and improved their ability to take corrective actions during emergencies. This will further improve operational safety and helps them to gain more confidence in executing the complex tasks. Over the course of time one can see the reduction of human errors due to repeated training and more than $30 \%$ of response's from the participants validate the same.

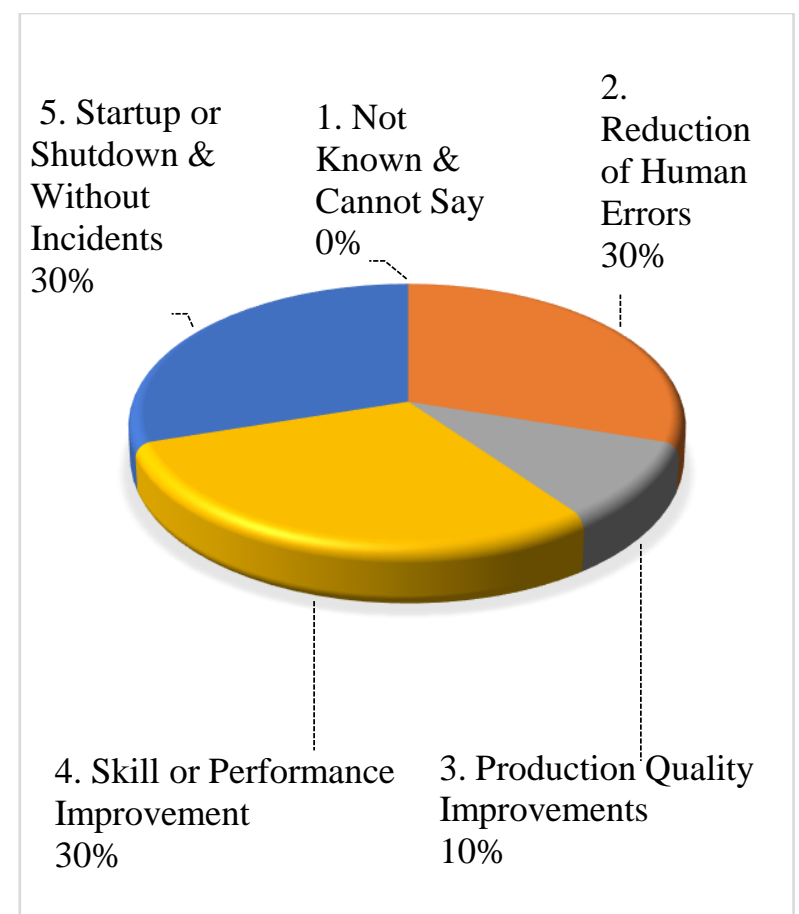

Figure 9. Supporting the OTS investment

\section{The percentage of improvement due to simulator training}

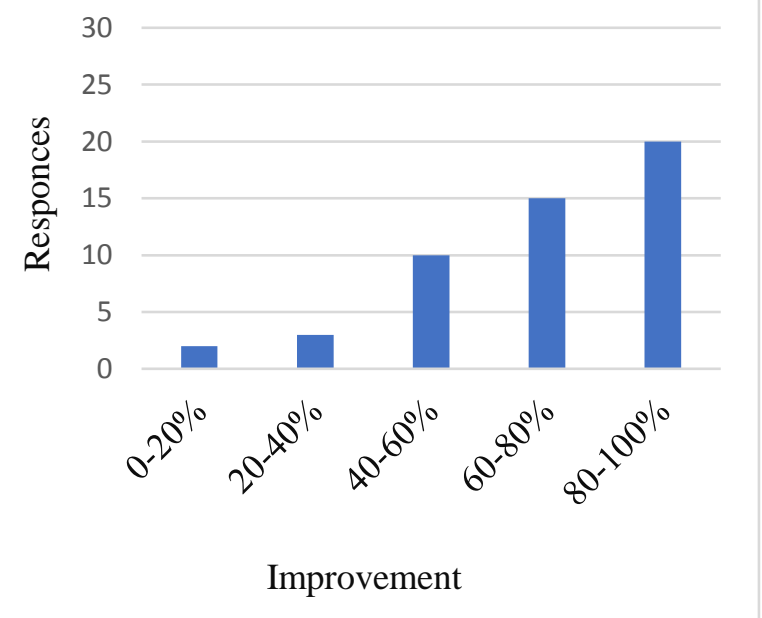

Figure 10. Results level findings

More than $80 \%$ of the participants, as per Figure 10. agrees that there is significant improvement in actual handling of operations after the training. The knowledge acquired from the training is effectively used at the work place and the OTS training is the most effective training across the industry to the operators. 
Based on the findings, we can conclude that the simulator training enhance operator skills, behaviour , performance, helped to reduce downtime, faster and more efficient startup and finally improve overall competency of staff.

\section{Limitations, Conclusions \& Future Outlook}

The survey is limited to a small group of participants and is relatively small size for proper statistical analysis. The results are analyzed using the data captured before and immediately after the training. Post training survey data after a certain period of time is not considered due to very low response for emails sent to the individuals. Further the analysis is not capturing the capability of the OTS process models in executing the tasks and the competency of instructors.

The simulator can be used to verify the procedures and they can then be optimized, rewritten to insist on critical points. The operators are confident in executing the complex tasks, leading to product quality, skill improvement and one might see a reduction in human errors due to the competency based approach. By integrating the training evaluation models with the OTS, the instructor can easily measure each and every session without being depend on questionnaires and infer the results. This methodology can optimize the trainings delivered and improve future sessions.

A combination of skilled instructors, immersive simulation, cloud technologies coupled with up-todate high-fidelity simulators and pedagogical course material are essential for ensuring the best possible training for the new and experienced operators in the oil and gas industry. The operators have to take objective based decisions based on real time data, strive to contribute process changes, and needs to be competent for the ever changing new cyber security protocols. The instructor role is changing due to new virtual \& augmented technologies, automated and objective based decisions. They also need to be trained to update with the technology, which is often missing due to various organizational reasons. There are number of challenges in the development of exceptional workforce to sustain the business. Staggered industrial growth, retiring and ageing workforce, an influx of new workers forcing the organizations to get new hires quickly trained and competent in the current low oil price environment and simulation training continues to be the best among the available trainings for operators and most cost effective way.

\section{References}

ARC Insights-2007, T. Fiske, ARC Advisory Group, Maine. (www.arcweb.com/market-studies/)

Bishop, R.L. Herron, 2015. Use and misuse of the Likert item responses and other ordinal measures.

Down, D.F., \& R. Mercer. "Applying marine simulation system to improve mariner professional development". PIANC, Port `95, TAMPA Florida, March 1995.

E. Salas., 2006. Design, delivery and evaluation of training systems. Handbook of human factors and ergonomics. Hoboken, NJ: Wiley and Sons, 472-512.

G. Tichon Jennifer \& Guy M. Wallis, Stress training and simulator complexity, why sometimes more is less by, The School of Human Movement Studies, University of Queensland, Australia.

Meshkati, International Journal of Occupational Safety and Ergonomics (JOSE) - 2006, Vol. 12, No. 1, Page 79-93.

Raja-2010, Complacency and Bias in Human Use of Automation: DOI: 10.1177/0018720810376055.

Smith, 1983. Sampling Considerations in Evaluating Cooperative Extension Programs, University of Florida.

Surender kumar, Effectiveness of seafarers training, Doctoral thesis-2015, COMES, LSCM, UPES, Dehradun, India.

Stetz. M., Wiederhold, B.K. and Wildzunas, R., 2006. Usefulness of virtual reality stress inoculation training for military medical females. Cyber psychology and Behaviour.

Taro Yamane, 1967. Statistics, an Introductory Analysis, 2nd Ed., New York: Harper and Row.

T. Bullemer Peter -1997, A New Training Strategy: Design the Work Environment for Continuous Learning, Honeywell Advanced Solutions centre.

Tichon et al., 2003. Proceedings of the World Conference on Educational Multimedia, Honolulu, Hawaii.

Winfred, Winston \& Pamela, Journal of Applied Psychology, The American Psychological Association Inc. 2003, Vol. 88, No. 2, Pages 234-245.

Wiener, 1989; Bainbridge, 1983; Wiener \& Curry, 1980, Flight-deck automation: promises and problems.

www.sciedu.ca/jnep (Designing the simulation learning environment: Journal of Nursing Education and Practice)

www.aveva.com/engineer-procure-construct/simulationand-training/ operator-training-simulators

www.kirkpatrickpartners.com/Our-Philosophy/TheKirkpatrick-Model

www.taproot.com/ archives/699 \& 6410

Yamnill. S. \& McLean, G. N. (2001), Theories supporting transfer of training, Human Resource Development Quarterly, pages 195-208.

Y.F. Barnard, Veldhuis, G.J., \& Van Rooij, J.C.G.M., (2001), "Evaluation in practice: Identifying factors for improving transfer of training in technical domains", Studies in Educational Evaluation, 27:269-290. 